\title{
Downregulated expression of human leukocyte antigen class $I$ heavy chain is associated with poor prognosis in non-small-cell lung cancer
}

\author{
KAZUOMI ICHINOKAWA ${ }^{1}$, YOSHITSUGU NAKANISHI ${ }^{1}$, YASUHIRO HIDA ${ }^{2}$, TAKAHIRO TSUCHIKAWA ${ }^{1}$, \\ TATSUYA KATO $^{2}$, TOMOO ITOH ${ }^{3}$, MITSUHITO KAJI $^{4}$, KICHIZO KAGA $^{2}$ and SATOSHI HIRANO ${ }^{1}$ \\ Departments of ${ }^{1}$ Gastroenterological Surgery II, and ${ }^{2}$ Cardiovascular and Thoracic Surgery, Hokkaido University \\ Graduate School of Medicine, Sapporo, Hokkaido 060-8638; ${ }^{3}$ Department of Surgical Pathology, Hokkaido University \\ Hospital, Sapporo, Hokkaido 060-8648; ${ }^{4}$ Department of Thoracic Surgery, Sapporo Minami Sanjo Hospital, \\ Sapporo, Hokkaido 060-0063, Japan
}

Received June 13, 2016; Accepted March 1, 2019

DOI: $10.3892 / \mathrm{ol} .2019 .10293$

\begin{abstract}
The aim of this study was to clarify the association between expression of human leukocyte antigen (HLA) class I in non-small-cell lung cancer (NSCLC) cells and patient survival. To address this, immunohistochemical staining for HLA class I was performed on specimens from 111 patients with NSCLC, and overall survival curves were compared using the log-rank test. In addition, multivariate analyses were performed using Cox's proportional hazard model. The cases were divided into 5 classes based on the expression of HLA class I heavy chain and $\beta 2$-microglobulin. The overall survival rate for patients with tumors lacking HLA class I heavy chain (30 cases; $27.0 \%$ ) was significantly decreased. The multivariate analysis demonstrated that the absence of HLA class I heavy chain was an independent predictor of poor prognosis. There was a trend towards an unfavorable prognosis for patients whose tumors did not express $\beta 2$-microglobulin (57 cases; 51.4\%). Downregulation of HLA class I heavy chain expression was significantly associated with the downregulation of $\beta 2$-microglobulin. Cases lacking HLA class I heavy chain as well as $\beta 2$-microglobulin expression (23 cases; $20.7 \%$ ) had a statistically significant unfavorable prognosis compared with other cases. The present findings demonstrate that the lack of HLA class I heavy chain expression in tumor cells is an independent prognostic factor for poor NSCLC survival, and is likely to exert an important influence on immune surveillance in patients.
\end{abstract}

Correspondence to: Dr Yoshitsugu Nakanishi, Department of Gastroenterological Surgery II, Hokkaido University Graduate School of Medicine, North 15, West 7, Kita-ku, Sapporo, Hokkaido 060-8638, Japan

E-mail: y.nakanishi@mac.com

Key words: human leukocyte antigen class I, $\beta 2$-microglobulin antigen, non-small-cell lung cancer, immunohistochemistry

\section{Introduction}

The most effective treatment for early stage non-small-cell lung cancer (NSCLC) is surgical resection. However, $>60 \%$ of patients with NSCLC relapse following surgery (1-3). The immune system can not only suppress tumor growth by destroying cancer cells or inhibiting their proliferation, but can also promote tumor progression by selecting for tumor cells that can survive in an immunocompetent host or by establishing conditions within the tumor microenvironment that facilitate tumor growth $(4,5)$. The anti-tumor arsenal of the immune system includes cluster of differentiation (CD) $4^{+}$ $\mathrm{T}$ cells that kill tumor cells in a major histocompatibility complex (MHC) class II expression-dependent manner, and natural killer cells/macrophages that mediate attacks on cells by antibody-dependent cellular cytotoxicity. In addition, $\mathrm{CD} 8^{+}$ $\mathrm{T}$ cells are hypothesized to serve an important role in the elimination of tumor cells by identifying human leukocyte antigen (HLA) class I expression on their surface. The association between HLA class I expression on tumor cells and CD8 ${ }^{+}$ $\mathrm{T}$ cell infiltration has been reported in laryngeal squamous (6), pancreatic (7) and ovarian carcinoma (8).

HLA class I molecules are transmembrane glycoproteins comprising a polymorphic $45-\mathrm{kDa}$ heavy chain and a non-polymorphic $12-\mathrm{kDa} \beta 2$-microglobulin light chain. There are 3 HLA class I heavy chains, HLA-A, -B and -C, encoded by 3 separate genes on chromosome $6 \mathrm{p} 21$. Loss or downregulation of HLA class I has been reported in various types of cancer, including pancreatic, ovarian and breast carcinoma (7-13), and a number of studies have described HLA class I downregulation in NSCLC (14-19). However, in these previous studies, there were discrepant associations between HLA class I downregulation and prognosis (15-17,19), which may be due to the use of different antibodies, control methods and tumor classification schemes. Attempts to standardize methods have included the use of the EMR8-5 antibody, which recognizes HLA-A, -B and -C (20), and the establishment of a novel control method for staining, using severe combined immune deficiency (SCID) mouse xenografts to examine the expression of CD40 and CD154 in NSCLC (21). 
However, a classification system based on immunostaining for loss or downregulation of HLA class I has not been established.

In the present study, the influence of downregulation of HLA class I in tumors on the survival of patients with NSCLC was assessed using EMR8-5 immunostaining, SCID mouse xenografts as quantitative controls and novel classification schemes based on tumor heterogeneity, and the association between loss or downregulation of HLA class I expression and NSCLC prognosis was reviewed.

\section{Materials and methods}

Cell lines. Human lung cancer cell lines were obtained from the Japanese Cancer Research Resources Bank (Tokyo, Japan). A549, H226, LC-1, LK2, PC3, PC10 and RERF-LC-MS cells were cultured in RPMI-1640 medium (Sigma-Aldrich; Merck KGaA, Darmstadt, Germany) with $10 \%$ fetal bovine serum (FBS; Sigma-Aldrich; Merck KGaA) and $1 \%$ penicillin/streptomycin. ABC-1 and VMRC-LCD cells were maintained in Eagle's Minimum Essential Medium (Sigma-Aldrich; Merck KGaA) with $10 \%$ FBS and $1 \%$ penicillin/streptomycin. All cell lines were maintained at $37^{\circ} \mathrm{C}$ in a humidified incubator with $5 \% \mathrm{CO}_{2}$. Peripheral blood cells were taken from a healthy volunteer and human peripheral blood mononuclear cells (PBMCs) were obtained using the Ficoll separation method.

Mice and xenograft models. Formalin-fixed paraffinembedded tissue specimens were obtained from mice xenograft models established in a previous study (22). CB17/SCID mice were obtained from Charles River Laboratories, Inc. (Wilmington, MA, USA). All mice were 4-6-week-old females and housed with food and water ad libitum under specific pathogen free conditions. The room was kept on a 12/12-h light/dark cycle, at $22.0-24.0^{\circ} \mathrm{C}$. Humidity was not regulated. All animal procedures were conducted in accordance with the guidelines of the Hokkaido University Institutional Animal Care and Use Committee using an approval protocol.

A total of 6 mice per one cell line (54 in total) were used. Xenografts from the lung cancer cell lines were generated by injecting $5 \times 10^{6}$ cells in $200 \mu \mathrm{l}$ PBS subcutaneously into the flanks of the CB17/SCID mice. When the tumor diameter exceeded $10 \mathrm{~mm}$, the mice were sacrificed and the tumors were separated into 2 portions. One portion was snap frozen using liquid nitrogen to extract proteins for western blot analysis, and the other was immersed in $10 \%$ formalin and incubated at $4^{\circ} \mathrm{C}$ for $24 \mathrm{~h}$ for immunohistological analysis.

Reagents and antibodies. The pan anti-human HLA-A, -B, and -C antigen mouse EMR8-5 monoclonal antibody (cat. no. FA01) and anti- $\beta 2$-microglobulin mouse EMRB6-12 monoclonal antibody (cat. no. GB-01B) were purchased from Hokudo Co., Ltd. (Sapporo, Japan). Negative control mouse IgG1 (cat. no. X0931) was purchased from Dako (Dako; Agilent Technologies, Inc., Santa Clara, CA, USA). Peroxidase-conjugated goat anti-mouse IgG (cat. no. 115-035-003) was purchased from Jackson ImmunoResearch Laboratories, Inc. (West Grove, PA, USA). Anti $\beta$-actin mouse antibody (cat. no. MAB 150-1R; Chemicon International; Thermo Fisher Scientific, Inc., Waltham, MA, USA) was used as the loading control of western blot analysis.
Western blot analysis. Western blotting was used to analyze the expression of HLA class I heavy chain and 32 -microglobulin in lung cancer cells. Each frozen tissue was crushed to powder in liquid nitrogen using a CRYO-PRESS (Microtec Co., Ltd., Chiba, Japan) and resuspended in radioimmunoprecipitation assay lysis buffer (Santa Cruz Biotechnology, Inc., Dallas, TX, USA) with the addition of protease inhibitors (Promega Corporation, Madison, WI, USA) and homogenized two times by Bioruptor (Cosmo Bio, Tokyo, Japan) for $30 \mathrm{sec}$ on ice. Lysates were incubated at $4^{\circ} \mathrm{C}$ for $20 \mathrm{~min}$ and centrifuged at $12,000 \mathrm{x} \mathrm{g}$ at $4^{\circ} \mathrm{C}$ for $20 \mathrm{~min}$ to remove cellular debris. Supernatants were recovered and protein concentrations were determined by a Bradford protein assay (Bio-Rad Laboratories, Inc., Hercules, CA, USA). Lysates (10 $\mu \mathrm{g}$ protein/lane) were resolved using 15\% SDS-PAGE, and then transferred to nitrocellulose membranes (GE Healthcare, Chicago, IL, USA). After blocking in TBS-Tween-20 (TBS-T; $50 \mathrm{mM}$ Tris, $150 \mathrm{mM} \mathrm{NaCl}, 0.01 \%$ Tween-20) with 5\% skimmed milk at room temperature for $1 \mathrm{~h}$, membranes were incubated with EMR8-5 (1:1,000 dilution) or EMR-B6 (1:400 dilution) antibodies at room temperature for $1 \mathrm{~h}$. The membrane was washed three times with TBS-T, 5 min each, and then incubated with the peroxidase-conjugated goat anti-mouse IgG secondary antibody (1:10,000 dilution) at room temperature for $1 \mathrm{~h}$. The bound antibodies were detected using enhanced chemiluminescence western blotting detection reagent (GEHealthcare Life Sciences, Shanghai, China) and ChemiDoc $\mathrm{XRS}+$ (Bio-Rad Laboratories, Inc.). Membranes were stripped of EMR 8-5, or EMR-B6 antibodies using Restore ${ }^{\mathrm{TM}}$ PLUS Western Blot Stripping Buffer (Thermo Fisher Scientific, Inc.), and probed with the anti $\beta$-actin antibody (1:10,000 dilution) at room temperature for $1 \mathrm{~h}$. Lysate from normal PBMCs was used as the positive control for HLA class I heavy chain and $\beta 2$-microglobulin expression.

Patients and tissue specimens. Whole surgical specimens of NSCLC obtained between December 1992 and January 2001 and relevant patient data were used in the present study. All patients lacked any evidence of metastases to secondary sites and had not received prior anticancer treatment. Adjacent matching normal tissue $>5 \mathrm{~cm}$ away from the tumor was additionally obtained and stored at $-80^{\circ} \mathrm{C}$. A section of the normal tissue was stained with hematoxylin $\&$ eosin (as described in the immunohistochemistry section) to verify there were no cancerous cells present. Cases of in-hospital and non-cancer-associated mortalities were excluded. Patients provided informed consent, and the study was conducted in accordance with the guidelines of The Hokkaido University Institutional Review Board (Sapporo, Japan). The study included a total of 111 NSCLC surgical specimens from patients meeting these criteria who had undergone curative resection of the primary tumor, including systematic lymph node dissection, at The Hokkaido University Hospital. The surgical specimens were obtained from 72 men and 39 women with a mean age of 62.7 years (range, 36-80 years). The median duration of follow-up was 60.9 months (range, 4.0-100.7 months), and 43 patients (38.7\%) succumbed during the follow-up period.

The clinical patient data were analyzed according to the expression results of their tissue samples. According to the 
World Health Organization classification system (23), the specimens consisted of 56 adenocarcinomas, 39 squamous carcinomas, 3 adenosquamous carcinomas, 12 large-cell carcinomas and 1 carcinosarcoma. The site and size of the lesion were assessed on the gross specimen. The clinicopathological stage of the tumor was determined according to the standard Tumor-Node-Metastasis (TNM) classification system (24). The results indicated that 50 samples were $\mathrm{T} 1$ and 61 were T2-4. The lymph nodes were negative in 79 patients and positive in 32. The stage of the surgical specimen was defined according to the 7th International Union Against Cancer Classification (UICC) (23). Diagnosis was performed independently by $\geq 2$ pathologists.

Immunohistochemistry. The surgical specimens were fixed in $10 \%$ formalin solution and kept at room temperature for 2-4 days. Subsequently, the specimens were embedded in paraffin for sectioning at $4 \mu \mathrm{m}$. The sections were then deparaffinized in xylene, dehydrated through a graded ethanol series and autoclaved for $5 \mathrm{~min}$. Endogenous peroxidase activity was blocked by a 10-min incubation with hydrogen peroxide at room temperature. Following 3 washes in PBS containing $0.1 \%$ Tween 20 , the sections were incubated in $10 \%$ normal goat serum (Histofine SAB-PO kit; Nichirei Biosciences, Inc., Tokyo, Japan) for $30 \mathrm{~min}$ at room temperature. The samples were then incubated at room temperature for $1 \mathrm{~h}$ with the EMR8-5 anti-human HLA class I heavy chain mouse monoclonal antibody (1:400 dilution). In addition, serial tissue sections of each sample were individually incubated at room temperature for $1 \mathrm{~h}$ with the EMR-B6 anti- $\beta 2$-microglobulin mouse monoclonal antibody (1:400 dilution). Following 3 additional washes, the sections were incubated with Histofine Simple Stain MAX-PO (Multi; Nichirei Biosciences, Inc.) for $30 \mathrm{~min}$ at room temperature and the reaction products were visualized by incubation for $\sim 10 \mathrm{~min}$ with 3,3'-diaminobenzidine tetrahydrochloride (Nichirei Biosciences, Inc.), followed by washing in distilled water. The sections were counterstained in hematoxylin for $1.5 \mathrm{~min}$ at room temperature, and mounted in Permount (Micro Slides; Muto Pure Chemicals Co., Ltd., Tokyo, Japan). The categories and percentages of immunohistochemical stained cells were assessed in five independent high-power (magnification, x400) microscopic fields for each tissue sample using a light microscope.

Statistical analysis. The statistical significance of associations between HLA class I heavy chain and $\beta 2$-microglobulin expression in cancer cells was evaluated using the Mann-Whitney $U$ test. The $\chi^{2}$ test (or extended Fisher's exact test, where appropriate) was used to analyze the comparisons between HLA class I heavy chain or $\beta 2$-microglobulin expression in cancer cells and patient parameters, including histopathological findings. The cumulative survival rate was calculated using the Kaplan-Meier method and statistical significance was analyzed with the log-rank test. Cancer-specific survival rates were calculated from date of surgery to date of cancer-associated mortality. Univariate and multivariate analyses were performed using the Cox proportional hazard regression model. $\mathrm{P}<0.05$ was considered to indicate a statistically significant difference. All analyses were performed using StatView-J version 5.0 software (SAS Institute, Inc., Cary, NC, USA).

\section{Results}

Expression of HLA class I heavy chain and $\beta 2$-microglobulin in NSCLC cell lines and their derived xenografts. Western blot analysis revealed that HLA class I heavy chain $(45 \mathrm{kDa})$ was detected most strongly in the H226 cell line (Fig. 1A; top panel), and expression was lowest in the VMRC-LCDand LK2-derived xenografts (Fig. 1A; lower panel). $\beta 2$-microglobulin (12 kDa) was strongly expressed in 4 cell lines: PC3, RERF-LC-MS, VMRC-LCD and H226 (Fig. 1B; top panel), and downregulated in 4 xenografts: ABC-1, VMRC-LCD, LC-1 and LK2 (Fig. 1B; bottom panel). HLA class I heavy chain was expressed in normal human lung tissue at levels similar to those detected in the PBMC (Fig. 1A; top panels). Of the 9 cell lines, A549 and PC10 demonstrated downregulated HLA class I heavy chain and $\beta 2$-microglobulin expression, although the xenografts from these cells exhibited higher expression of these molecules. PC10-derived xenografts were used as positive tissue controls for HLA class I heavy chain and $\beta 2$-microglobulin expression because the proteins were highly expressed and clearly detected in these samples (Figs. 1A, 2A and B). LK2-derived xenografts, which exhibited downregulated HLA class I heavy chain and $\beta 2$-microglobulin expression by western blotting, were used as negative tissue controls (Figs. 1B, 2C and D). From these results, the xenografts derived from $\mathrm{PC} 10$ and LK2 cells were used as positive and negative controls, respectively, for HLA class I expression. Isotype-matched negative control antibodies were used to control for non-specific staining.

Establishment of a quantitative evaluation for tumor HLA class I and $\beta 2$-microglobulin expression. Immunostaining intensities were grouped into 3 categories: Strongly positive (equivalent to staining of alveolar epithelium), weakly positive relative to alveolar epithelium, and absent (no detectable staining) (Fig. 2).

Expression of HLA class I heavy chain and $\beta 2$-microglobulin in tumors of patients with NSCLC. The mean of five independent areas occupied by each of these three immunostaining intensity categories (strongly positive, weakly positive, or absent) of HLA class I heavy chain and $\beta 2$-microglobulin in each of the cases was calculated and graded in $10 \%$ steps. The cases were further divided into 5 categories: Homogeneous strong staining (Group A), heterogeneous strong/weak staining (Group B), homogeneous weak staining (Group C), heterogeneous strong/weak/absent staining (Group D) and heterogeneous weak/absent staining (Group E).

Regarding the HLA class I heavy chain staining, of the 111 cases, 31 (27.9\%) were classified into Group A, 38 (34.2\%) into Group B, 12 (10.8\%) into Group C, 21 (18.9\%) into Group D and 9 (8.1\%) into Group E (Fig. 3A). With regards to $\beta 2$-microglobulin staining, 12 tumors $(10.8 \%)$ were classified into Group A, 37 (33.3\%) into Group B, 5 (4.5\%) into Group C, $48(43.2 \%)$ into Group D and 9 (8.1\%) into Group E (Fig. 3B).

Survival analysis and HLA class I heavy chain and $\beta 2$-microglobulin expression. The 5-year disease-specific survival rate was $62.2 \%$ for all patients (data not shown). Kaplan-Meier survival curves for each HLA class I heavy chain 

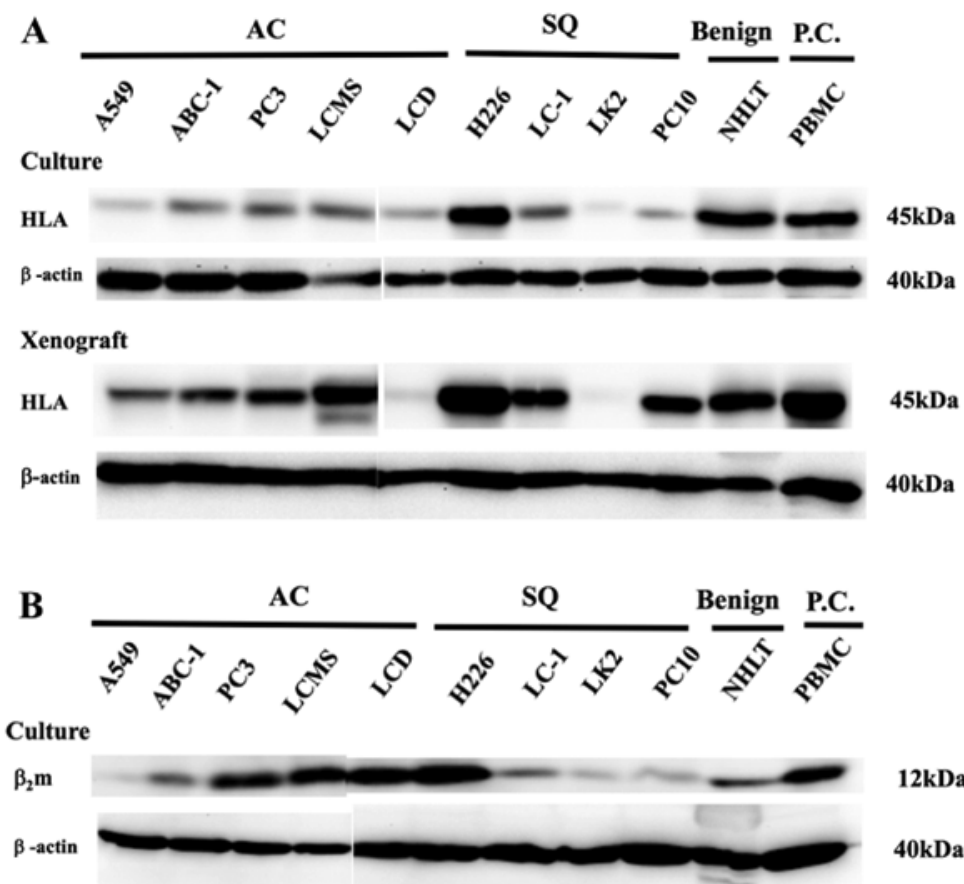

$12 \mathrm{kDa}$

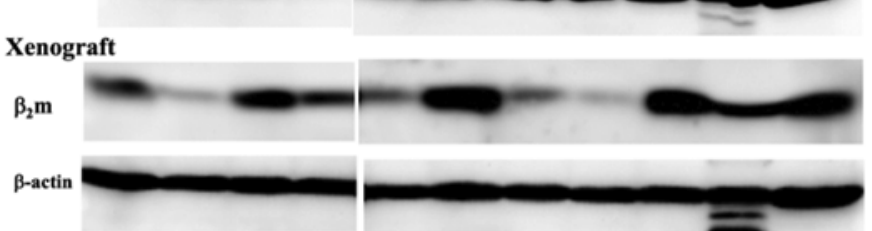

$40 \mathrm{kDa}$

$12 \mathrm{kDa}$

40kDa

Figure 1. Representative images of western blot analyses for (A) HLA and (B) $\beta 2 \mathrm{~m}$ in human lung cancer cell lines (top panel) and lysates from mouse xenograft models constructed using the lung cancer cell lines (bottom panel). Lysates from homogenized NHLT and human PBMCs were used as non-tumor control and PC, respectively. HLA, human leukocyte antigen class I heavy chain; $\beta 2 \mathrm{~m}, \beta 2$-microglobulin; LCMS, RERF-LC-MS; LCD, VMRC-LCD; AC, adenocarcinoma; SCC, squamous cell carcinoma; NHLT, normal human lung tissue; PBMCs, peripheral mononuclear cells; PC, positive control.

\section{PC10}

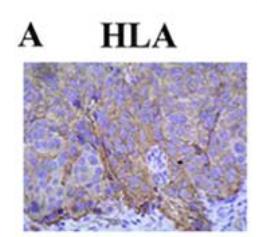

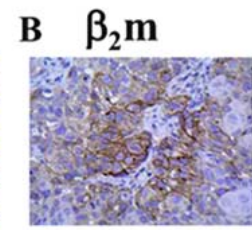

\section{LK2}

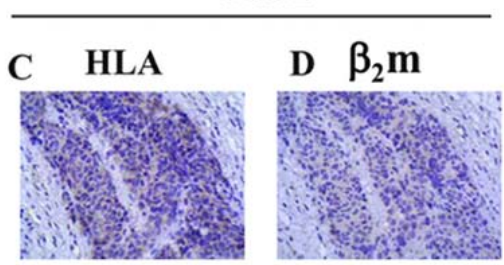

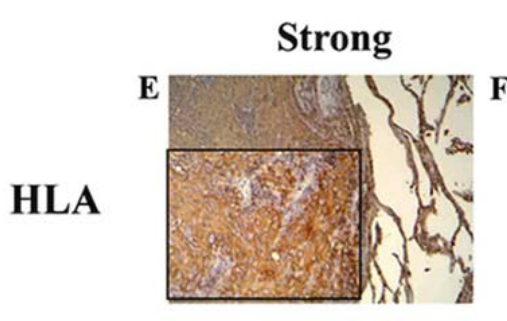
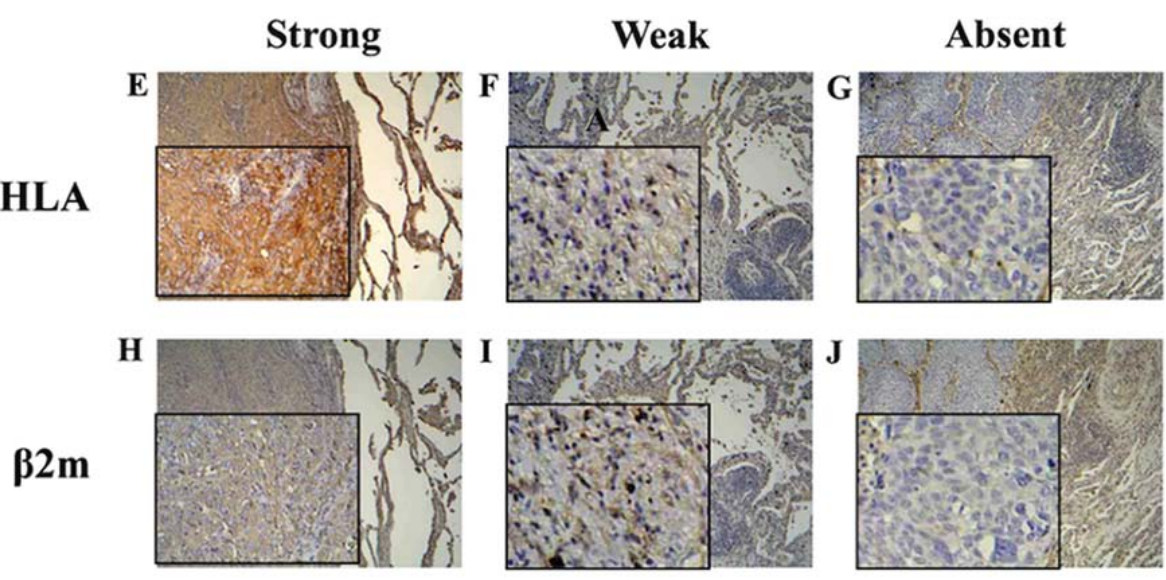

Figure 2. Immunohistochemical staining for HLA and $\beta 2 \mathrm{~m}$. The top panel includes images of HLA and $\beta 2 \mathrm{~m}$ staining in mouse xenograft specimens. Expression of (A) HLA or (B) $\beta 2 \mathrm{~m}$ in PC10-derived tumor. Absence of (C) HLA expression or (D) $\beta 2 \mathrm{~m}$ expression in LK2-derived tumor. Magnification, $\mathrm{x} 400$. The bottom two panels are images of non-small-cell lung cancer samples in which the cell membranes are stained for HLA or $\beta 2 \mathrm{~m}$. (E) Strong, (F) weak and (G) no staining for HLA. (H) Strong, (I) weak and (J) no staining for $\beta 2 \mathrm{~m}$. Larger panel magnification, x100; smaller panel magnification, x400. HLA, human leukocyte antigen class I heavy chain; $\beta 2 \mathrm{~m}, \beta 2$-microglobulin. 
A

Group of HLA class I heavy chain expression

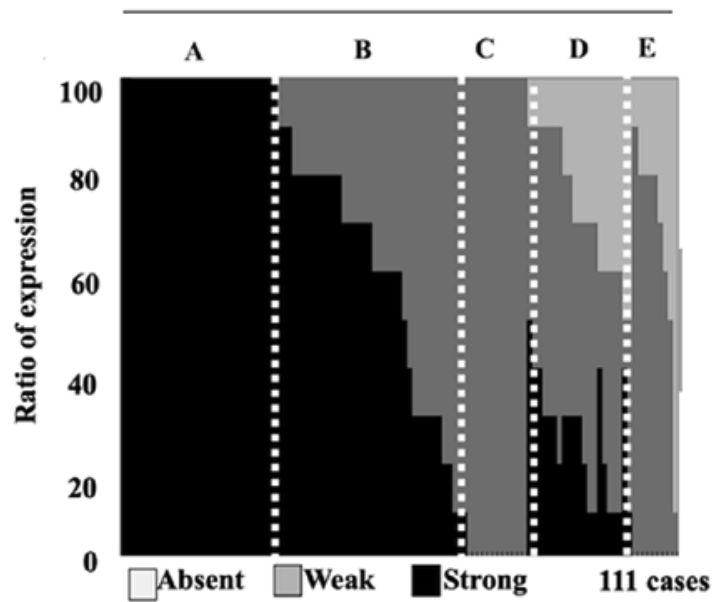

B Group of $\mathbf{B 2}$ microglobulin expression

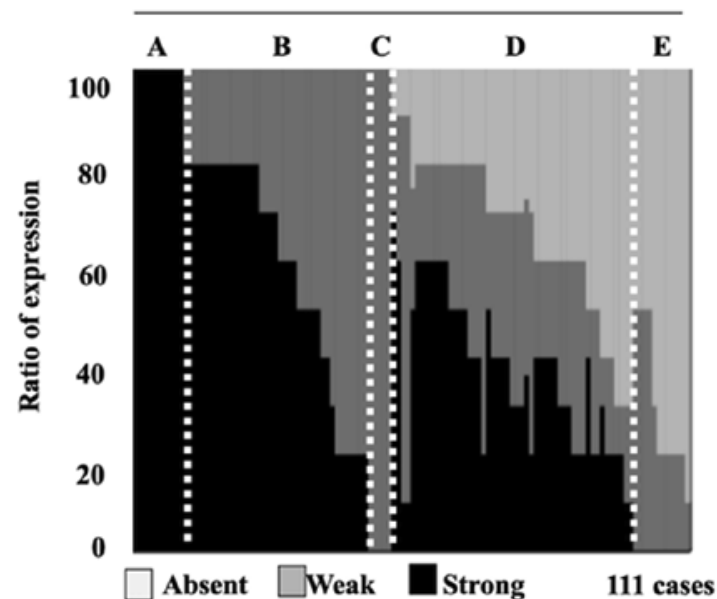

Figure 3. HLA class I heavy chain and $\beta 2$-microglobulin expressions. (A) HLA class I heavy chain expression in NSCLC. Group A, $\mathrm{n}=31$; group B, $\mathrm{n}=38$; group $\mathrm{C}, \mathrm{n}=12$; group $\mathrm{D}, \mathrm{n}=21$; and group $\mathrm{E}, \mathrm{n}=9$. (B) $\beta 2$-microglobulin expression in NSCLC. Group A, $\mathrm{n}=12$; group $\mathrm{B}$, $\mathrm{n}=37$; group C, $\mathrm{n}=5$; group $\mathrm{D}$, $\mathrm{n}=48$; and group $\mathrm{E}, \mathrm{n}=9$. HLA, human leukocyte antigen; NSCLC, non-small-cell lung cancer; group A, homogeneous strong staining; group B, heterogeneous strong/weak staining; group C, homogeneous weak staining; group D, heterogeneous strong/weak/absent staining; group E, heterogeneous weak/absent staining.

A

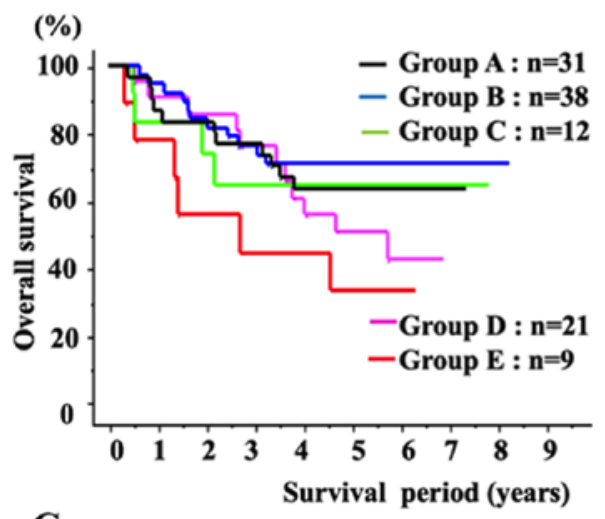

C

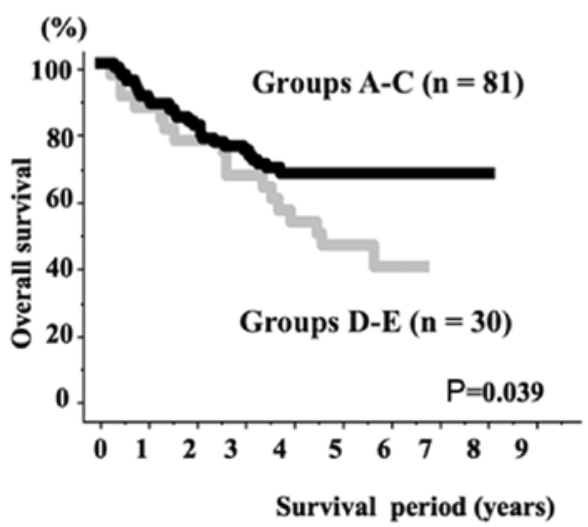

B

\begin{tabular}{|c|c|c|}
\hline Group & 5-year survival rate (\%) & P-value \\
\hline A vs. B-E & $63.4 \%$ vs. $60.4 \%$ & 0.735 \\
\hline A-B vs. C-E & $64.7 \%$ vs. $50.7 \%$ & 0.073 \\
\hline A-C vs. D-E & $67.1 \%$ vs. $45.1 \%$ & 0.039 \\
\hline A-D vs. E & $63.7 \%$ vs. $33.3 \%$ & 0.031 \\
\hline
\end{tabular}

D

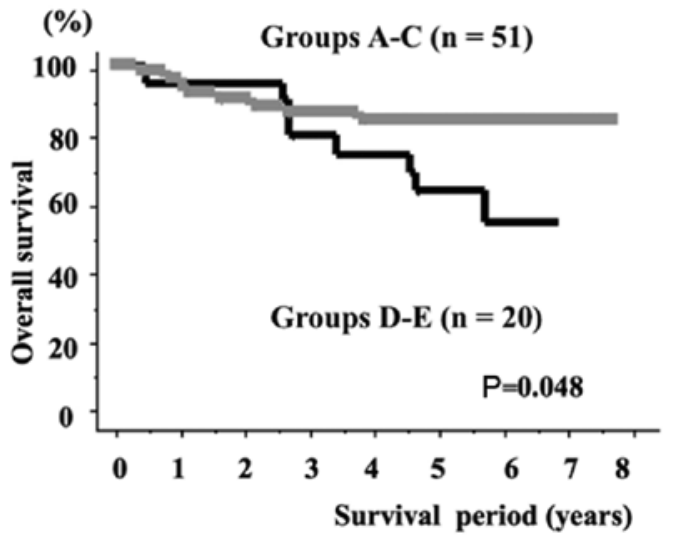

Figure 4. Survival analysis of HLA class I heavy chain. (A) Survival curves of patients with NSCLC divided into 5 groups according to the tumor HLA class I heavy chain expression. No significant differences in prognosis were observed among the groups. (B) Survival analyses where patients were divided into 2 groups according to expression of HLA class I heavy chain. (C) Survival curves of patients with NSCLC divided into 2 groups according to the HLA class I heavy chain expression, positive ( $\mathrm{n}=81$ ) and negative $(\mathrm{n}=30)$. (D) Survival curves of patients with stage I NSCLC according to their HLA class I heavy chain expression, positive $(\mathrm{n}=67)$ and negative $(\mathrm{n}=24)$. HLA, human leukocyte antigen; NSCLC, non-small-cell lung cancer; group A, homogeneous strong staining; group B, heterogeneous strong/weak staining; group C, homogeneous weak staining; group D, heterogeneous strong/weak/absent staining; group E, heterogeneous weak/absent staining.

expression group and for intergroup comparisons are plotted in Fig. 4A. The overall prognosis of groups without an absent part of HLA class I heavy chain expression in tumors (Group
A-C) was favorable compared with the groups with absent parts of HLA class I heavy chain expression (Group D-E; $\mathrm{P}=0.039$; Fig. 4B and C). When divided according to UICC 7th cancer 
$\mathbf{A}$

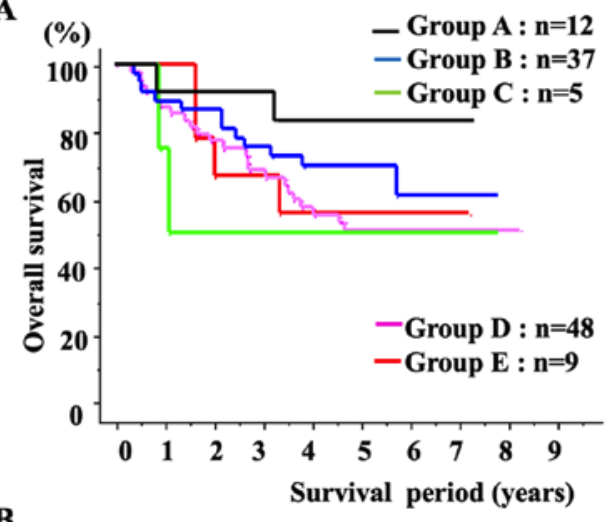

B

\begin{tabular}{|c|c|c|}
\hline Group & 5-year survival rate (\%) & P-value \\
\hline A vs. B-E & $83.3 \%$ vs. $58.4 \%$ & 0.114 \\
\hline A-B vs. C-E & $\mathbf{7 3 . 1} \%$ vs. $\mathbf{5 1 . 5 \%}$ & 0.057 \\
\hline A-C vs. D-E & $71.5 \%$ vs. $51.5 \%$ & 0.092 \\
\hline A-D vs. E & $61.7 \%$ vs. $55.6 \%$ & 0.777 \\
\hline
\end{tabular}

Figure 5. Survival analysis of $\beta 2$-microglobulin expression. (A) Survival curves of patients with NSCLC divided into 5 groups according to tumor $\beta 2$-microglobulin expression. No significant differences in prognosis were observed among the groups. (B) Survival analyses where patients were divided into 2 groups according to expression of $\beta 2$-microglobulin. NSCLC, non-small-cell lung cancer; group A, homogeneous strong staining; group B, heterogeneous strong/weak staining; group $\mathrm{C}$, homogeneous weak staining; group D, heterogeneous strong/weak/absent staining; group E, heterogeneous weak/absent staining.

stage, in 71 cases with stage I, 51 cases in Group A-C had a favorable prognosis compared with 20 cases in Group D-E $(\mathrm{P}=0.048$; Fig. 4D), but no significant difference in survival rate was observed between the groups of stages II-III $(\mathrm{P}=0.104$; data not shown).

The survival curves according to $\beta 2$-microglobulin expression groups are plotted in Fig. 5A. No significant difference in survival rate was observed between the groups overall (Fig. 5B).

Associations between HLA class I heavy chain and $\beta 2$-microglobulin according to immunostaining expression patterns. From the results of survival analysis of HLA class I heavy chain expressions, Group A-C was defined as the 'positive' group and Group D-E as the 'negative' group in both of HLA class I heavy chain and $\beta 2$-microglobulin in the present study (Table I). When HLA class I heavy chain and $\beta 2$-microglobulin were analyzed together, 46 tumors (41.4\%) were double positive, $35(31.5 \%)$ were positive only for HLA class I heavy chain, $8(7.2 \%)$ were positive only for the $\beta 2$-microglobulin antigen and $22(19.8 \%)$ were double negative. Additionally, a significant association between the expression of HLA class I heavy chain and $\beta 2$-microglobulin was observed $(\mathrm{P}=0.005)$.

Tumor HLA class I heavy chain expression and clinicopathological features. The tumor HLA class I heavy chain
Table I. Tumor HLA class I heavy chain expression and clinicopathological variables.

\begin{tabular}{|c|c|c|c|}
\hline \multirow[b]{2}{*}{ Variables } & \multicolumn{2}{|c|}{$\begin{array}{l}\text { HLA class I heavy } \\
\text { chain staining, } \mathrm{n}\end{array}$} & \multirow[b]{2}{*}{$\mathrm{P}$-value } \\
\hline & $\begin{array}{l}\text { Negative } \\
(n=30)\end{array}$ & $\begin{array}{l}\text { Positive } \\
(n=81)\end{array}$ & \\
\hline Age, years & & & 0.045 \\
\hline$\leq 62$ & 11 & 47 & \\
\hline$>62$ & 19 & 34 & \\
\hline Sex & & & 0.255 \\
\hline Male & 22 & 50 & \\
\hline Female & 8 & 31 & \\
\hline Histology & & & $0.312^{\mathrm{a}}$ \\
\hline $\mathrm{AC}$ & 13 & 43 & \\
\hline SQCC & 13 & 25 & \\
\hline $\mathrm{LCC}$ & 2 & 11 & \\
\hline Other & 2 & 2 & \\
\hline $\mathrm{T}$ classification & & & 0.825 \\
\hline pT1 & 13 & 37 & \\
\hline pT2-4 & 17 & 44 & \\
\hline Lymph node metastasis & & & 0.760 \\
\hline Absence & 22 & 57 & \\
\hline Presence & 8 & 24 & \\
\hline Histopathological grading & & & 0.835 \\
\hline G1 & 9 & 22 & \\
\hline $\mathrm{G} 2-3$ & 17 & 46 & \\
\hline TNM stage & & & 0.718 \\
\hline I & 20 & 51 & \\
\hline II-III & 10 & 30 & \\
\hline$\beta 2$-microglobulin staining & & & 0.005 \\
\hline Positive & 8 & 46 & \\
\hline Negative & 22 & 35 & \\
\hline
\end{tabular}

${ }^{\mathrm{a} C}$ Calculated using Fisher's exact test. AC, adenocarcinoma; SCC, squamous cell carcinoma; LCC, large-cell carcinoma; Other, adenosquamous carcinoma ( 3 cases) and carcinosarcoma ( 1 case); TNM, Tumor-Node-Metastasis.

expression results were analyzed against the clinicopathological data of the patient and no significant associations were detected between the expression levels and sex, histology, $\mathrm{T}$ classification, $\mathrm{N}$ classification, histopathological grading or TNM stage (Table I).

Univariate and multivariate analyses. Univariate analysis for overall survival using a Cox regression model included age, sex, $\mathrm{T}$ classification, $\mathrm{N}$ classification, TNM stage, histopathological grade and tumor expression of HLA class I heavy chain for the patient cohort included in this study. Multivariate analysis on the same set of patients was performed for $\mathrm{T}$ classification, $\mathrm{N}$ classification, and tumor expression of HLA class I heavy chain. The results indicated that absence of the HLA class I heavy chain is an independent prognostic factor 
Table II. Univariate and multivariate analysis of clinicopathological factors affecting overall survival of patients.

\begin{tabular}{lrrrrrrrr}
\hline & \multicolumn{3}{c}{ Univariate analysis } & & \multicolumn{2}{c}{ Multivariate analysis } \\
\cline { 2 - 3 } Variables & HR & $95 \%$ CI & P-value & & HR & $95 \%$ CI & P-value \\
\hline Age (>62 vs. $\leq 62)$, years & 1.77 & $0.97-3.25$ & & 0.065 & & & \\
Sex & 1.98 & $0.99-3.94$ & & 0.051 & & & \\
T-classification (1 vs. 2-4) & 4.08 & $2.00-8.33$ & $<0.001$ & & 3.48 & $1.70-7.14$ & 0.001 \\
N-classification & 4.17 & $2.25-7.69$ & $<0.001$ & & 3.72 & $1.99-6.94$ & $<0.001$ \\
TNM stage (I vs. II-III) & 4.61 & $2.45-8.62$ & $<0.001$ & & & \\
Histopathological grading (1 vs. 2-3) & 1.31 & $0.67-2.54$ & & 0.433 & & & \\
HLA class I heavy chain staining (negative vs. positive) & 1.88 & $1.02-3.47$ & & 0.043 & & 1.96 & $1.06-3.62$ & 0.032 \\
\hline
\end{tabular}

HR, hazard ratio; CI, confidence interval; TNM, Tumor-Node-Metastasis.

$(\mathrm{P}=0.032)$. Additionally, $\mathrm{T}$ and $\mathrm{N}$ classification had independent prognostic value, with hazard ratios of $3.48(\mathrm{P}=0.001)$ and $3.72(\mathrm{P}<0.001)$, respectively (Table II).

\section{Discussion}

The aim of the present study was to develop a quantitative immunohistochemistry protocol to investigate whether expression of HLA class I in NSCLC was associated with patient survival. Previously reported associations are controversial and difficult to compare because of differences in antibodies, positive controls and classification systems (Table III). A reproducible and carefully controlled quantitative immunohistochemistry method was developed, providing a robust approach for accurate classification of NSCLC specimens into categories based on HLA class I expression levels and characteristics. One of the most important issues in previous studies was a lack of consideration of tumor heterogeneity in immunohistochemistry evaluation. Therefore, in the present study, HLA class I downregulation was classified in consideration of tumor heterogeneity, assessed using EMR8-5 and SCID mouse xenografts. This methodology may be useful for others working in the field, and may provide a means to improve the consistency and comparability of similar studies in the future.

Whereas strong expression of HLA class I in the carcinoma cell membrane was easily distinguished from lack of expression, the categorization of tumors with weak expression was more challenging. To address this, the NSCLC cases were divided into 5 groups according to the HLA class I expression pattern. Furthermore, these 5 groups were classified into different combinations of 2 groups primarily based on strong or absent expression of HLA class I. The correlations between prognosis and the different combinations of groups were investigated. Groups of patients with absent expression of parts of HLA class I heavy chain demonstrated a significantly unfavorable prognosis compared with no absence of HLA class I. The present data may reflect the result of emerging immune-escaping variants, indicating the intra-tumor heterogeneity for HLA class I. However, although there were notable associations in immunostaining expressions with HLA class I heavy chain, $\beta 2$-microglobulin was not associated with the prognosis of patients with lung cancer in our cohort.
In the present study, the absence of HLA class I heavy chain expression on at least a part of the tumors (in Group D or E) was revealed to be an independent factor predicting poor prognosis, although expression of this factor was not associated with the tested clinicopathological variables. These results suggest that NSCLC would progress regardless of the expression of HLA class I heavy chain, and host immunity serves a limited role in influencing tumor growth prior to surgery. However, following surgery, relapse occurred in a higher number of cases with absent parts of HLA class I compared with cases with detectable levels of expression. Furthermore, the prognosis was particularly favorable for patients with cancer stage I and HLA class I heavy chain-positive cases, but in patients with advanced stage (II or III) cancer, no association was observed between prognosis and HLA class I heavy chain expression, in agreement with previous studies $(17,19)$. Therefore, the most suitable individuals for treatment with immune therapy for NSCLC may be patients with earlier stages of cancer.

It is well accepted that inflammation or an inflammatory gene expression in tumors is associated with anti-tumor immune responses $(25,26)$. MHC class I expression is predominantly upregulated by interferon- $\gamma$ in the tumor microenvironment. In addition, interferon- $\gamma$ is essential for eliciting the immune response to tumor cells (27). Therefore, the findings of the present study, which link HLA class I heavy chain expression with prognosis, may involve interferon- $\gamma$ signaling. Certain active immunotherapies, including novel agents capable of generating anti-tumor immunity, have been reported to exert clinical efficacy against lung cancer (28), and may act by boosting the interferon- $\gamma$ signature in the tumor microenvironment. Notably, immunological responses were independent of stage and prior therapy in a study of NSCLC dendritic cell vaccines (29), consistent with the possibility that the effectiveness of immunotherapy may be associated with expression of HLA class I heavy chain in tumor cells.

Currently, recurrent or surgically non-resectable tumors are often treated with immunotherapy, although the immune system of the patients has a limited capacity to deal with the heavy tumor burden. The results of the present study suggest that immunotherapy would be beneficial as postoperative adjuvant therapy in NSCLC. 


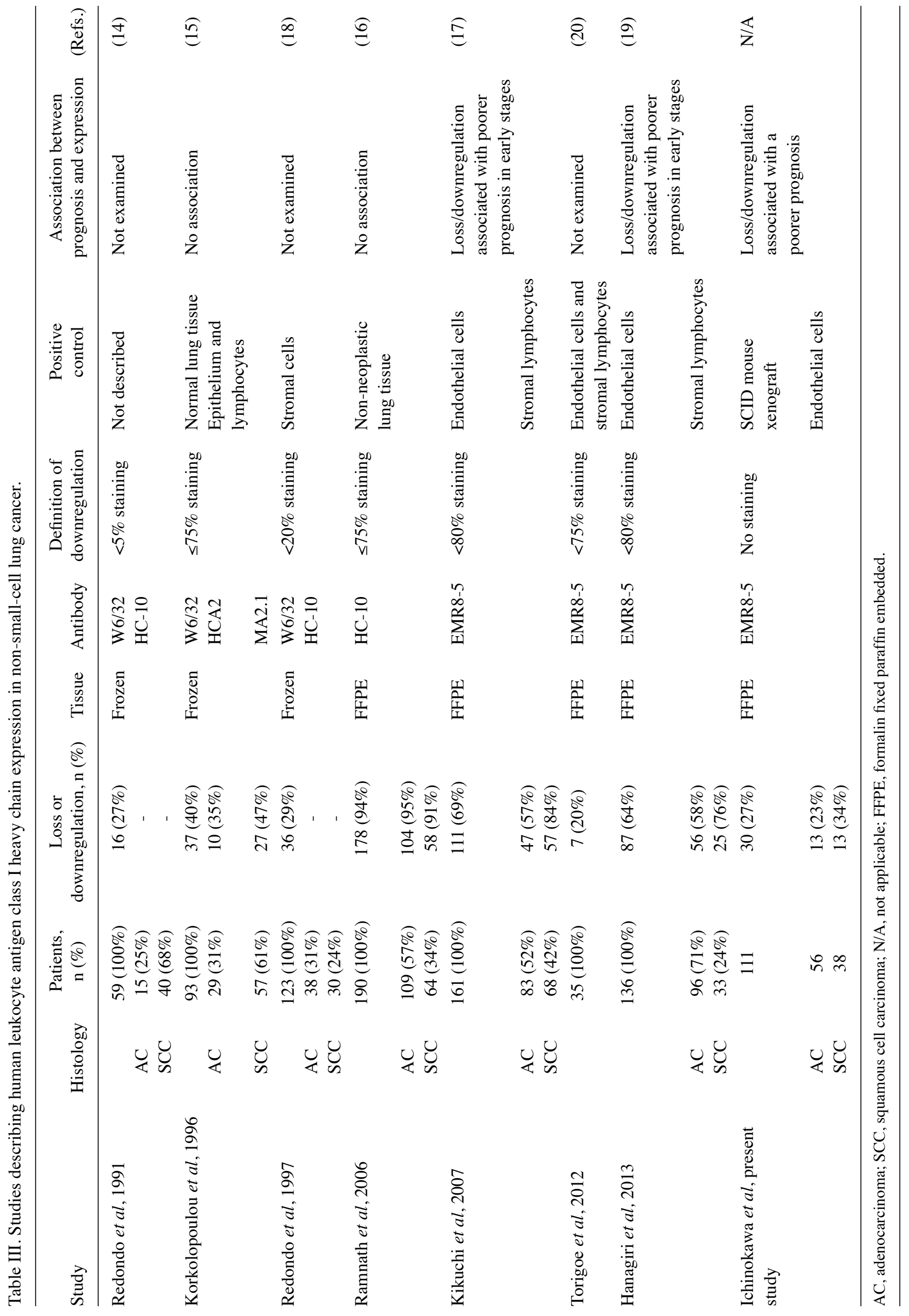




\section{Acknowledgements}

The authors would like to thank Mr Hiraku Shida and Ms Naomi Saito, The Affiliated Department of Gastroenterological Surgery II, Hokkaido University Graduate School of Medicine (Sapporo, Japan), for their technical assistance.

\section{Funding}

The present study was supported by a Grant-in-Aid from the Ministry of Education, Culture, Sports, Science and Technology, Japan (grant no. 18591534).

\section{Availability of data and materials}

The datasets used and/or analyzed during the present study are available from the author on reasonable request.

\section{Authors' contributions}

KI designed the present study, analyzed and interpreted the patient data, performed the histological examination, and was a major contributor in writing the manuscript. YN interpreted the data and drafted the manuscript. YH, TK and KK performed the surgical procedures and treated the specimens. TT interpreted the data and revised the manuscript. TI performed the histological examinations and evaluated the immunohistochemical specimens. MK performed the operations and treated the specimens. SH interpreted the data and drafted the manuscript.

\section{Ethics approval and consent to participate}

Patients provided informed consent to participate in the present study, which was approved by The Hokkaido University Institutional Review Board (approval no. 017-0476). Mice specimens used in this study were obtained from a previous study which was conducted in accordance with the guidelines of The Hokkaido University Institutional Animal Care and Use Committee using an approval protocol (approval no. 04157).

\section{Patient consent for publication}

Not applicable.

\section{Competing interests}

The authors declare that they have no competing interests.

\section{References}

1. Goya T, Asamura H, Yoshimura H, Kato H, Shimokata K, Tsuchiya R, Sohara Y, Miya T and Miyaoka E; Japanese Joint Committee of Lung Cancer Registry: Prognosis of 6644 resected non-small cell lung cancers in Japan: A Japanese lung cancer registry study. Lung Cancer 50: 227-234, 2005.

2. Mountain CF: Revisions in the international system for staging lung cancer. Chest 111: 1710-1717, 1997.

3. Sawabata N, Miyaoka E, Asamura H, Nakanishi Y, Eguchi K, Mori M, Nomori H, Fujii Y, Okumura M and Yokoi K; Japanese Joint Committee for Lung Cancer Registration: Japanese lung cancer registry study of 11,663 surgical cases in 2004: Demographic and prognosis changes over decade. J Thorac Oncol 6: 1229-1235, 2011.
4. Dunn GP, Bruce AT, Ikeda H, Old LJ and Schreiber RD: Cancer immunoediting: From immunosurveillance to tumor escape. Nat Immunol 3: 991-998, 2002.

5. Dunn GP, Old LJ and Schreiber RD: The three Es of cancer immunoediting. Annu Rev Immunol 22: 329-360, 2004.

6. Ogino T, Shigyo H, Ishii H, Katayama A, Miyokawa N, Harabuchi Y and Ferrone S: HLA class I antigen down-regulation in primary laryngeal squamous cell carcinoma lesions as a poor prognostic marker. Cancer Res 66: 9281-9289, 2006.

7. Ryschich E, Cebotari O, Fabian OV, Autschbach F, Kleeff J, Friess H, Bierhaus A, Büchler MW and Schmidt J: Loss of heterozygosity in the HLA class I region in human pancreatic cancer. Tissue Antigens 64: 696-702, 2004.

8. Kooi S, Zhang HZ, Patenia R, Edwards CL, Platsoucas CD and Freedman RS: HLA class I expression on human ovarian carcinoma cells correlates with T-cell infiltration in vivo and T-cell expansion in vivo and T-cell expansion in vitro in low concentrations of recombinant interleukin-2. Cell Immunol 174: 116-128, 1996.

9. Madjd Z, Spendlove I, Pinder SE, Ellis IO and Durrant LG: Total loss of MHC class I is an independent indicator of good prognosis in breast cancer. Int J Cancer 117: 248-255, 2005.

10. Watson NF, Ramage JM, Madjd Z, Spendlove I, Ellis IO, Scholefield JH and Durrant LG: Immunosurveillance is active in colorectal cancer as downregulation but not complete loss of MHC class I expression correlates with a poor prognosis. Int $\mathbf{J}$ Cancer 118: 6-10, 2006.

11. Kitamura H, Honma I, Torigoe T, Asanuma H, Sato N and Tsukamoto T: Down-regulation of HLA class I antigen is an independent prognostic factor for clear cell renal cell carcinoma. J Urol 177: 1269-1272, 2007.

12. Rolland P, Deen S, Scott I, Durrant L and Spendlove I: Human leukocyte antigen class I antigen expression is an independent prognostic factor in ovarian cancer. Clin Cancer Res 13: 3591-3596, 2007.

13. Jager MJ, Hurks HM, Levitskaya J and Kiessling R: HLA expression in uveal melanoma: There is no rule without some exception. Hum Immunol 63: 444-451, 2002.

14. Redondo M, Ruiz-Cabello F, Concha A, Cabrera T, Pérez-Ayala M, Oliva MR and Garrido F: Altered HLA class I expression in non-small cell lung cancer is independent of c-myc activation. Cancer Res 51: 2463-2468, 1991.

15. Korkolopoulou P, Kaklamanis L, Pezzella F, Harris AL and Gatter KC: Loss of antigen-presenting molecules (MHC class I and TAP-1) in lung cancer. Br J Cancer 73: 148-153, 1996.

16. Ramnath N, Tan D, Li Q, Hylander BL, Bogner P, Ryes L and Ferrone S: Is downregulation of MHC class I antigen expression in human non-small cell lung cancer associated with prolonged survival? Cancer Immunol Immunother 55: 891-899, 2006.

17. Kikuchi E, Yamazaki K, Torigoe T, Cho Y, Miyamoto M, Oizumi S, Hommura F, Dosaka-Akita H and Nishimura M: HLA class I antigen expression is associated with a favorable prognosis in early stage non-small cell lung cancer. Cancer Sci 98: 1424-1430, 2007.

18. Redondo M, Concha A, Ruiz-Cabello F, Morell M, Esteban F, Talavera P and Garrido F: Class I major histocompatibility complex antigens and tumor ploidy in breast and bronchogenic carcinomas. Cancer Detect Prev 21: 22-28, 1997.

19. Hanagiri T, Shigematsu Y, Kuroda K, Baba T, Shiota H, Ichiki Y, Nagata Y, Yasuda M, Uramoto H, So T, et al: Prognostic implications of human leukocyte antigen class I expression in patients who underwent surgical resection for non-small-cell lung cancer. J Surg Res 181: e57-e63, 2013.

20. Torigoe T, Asanuma H, Nakazawa E, Tamura Y, Hirohashi Y, Yamamoto E, Kanaseki T, Hasegawa T and Sato N: Establishment of a monoclonal anti-pan HLA class I antibody suitable for immunostaining of formalin-fixed tissue: Unusually high frequency of down-regulation in breast cancer tissues. Pathol Int 62: 303-308, 2012.

21. Ishikawa K, Miyamoto M, Yoshioka T, Kadoya M, Li L, Mishra R, Ichinokawa K, Shoji Y, Matsumura Y, Hida Y, et al: Method for the validation of immunohistochemical staining using SCID mouse xenografts: Expression of CD40 and CD154 in human non-small cell lung cancer. Oncol Rep 29: 1315-1321, 2013.

22. Ishikawa K, Miyamoto M, Yoshioka T, Kato T, Kaji M, Ohbuchi T, Hirano S, Itoh T, Dosaka-Akita $\mathrm{H}$ and Kondo S: Up-regulation of CD40 with juxtacrine activity in human nonsmall lung cancer cells correlates with poor prognosis. Cancer 113: 530-540, 2008. 
23. Sobin LH, Gospodarowicz MK and Wittekind C: International Union Against Cancer. TNM classification of malignant tumours 7th edition. Wiley-Liss, New York, 2009.

24. Travis WD, Brambilla E, Nicholson AG, Yatabe Y, Austin JHM, Beasley MB, Chirieac LR, Dacic S, Duhig E, Flieder DB, et al: The 2015 world health organization classification of lung tumors: Impact of genetic, clinical and radiologic advances since the 2004 classification. J Thorac Oncol 10: 1243-1260, 2015.

25. Spranger S, Spaapen RM, Zha Y, Williams J, Meng Y, Ha TT and Gajewski TF: Up-regulation of PD-L1, IDO, and T(regs) in the melanoma tumor microenvironment is driven by CD8(+) T cells. Sci Transl Med 5: 200ra116, 2013
26. Rooney MS, Shukla SA, Wu CJ, Getz G and Hacohen N: Molecular and genetic properties of tumors associated with local immune cytolytic activity. Cell 160: 48-61, 2015.

27. Shankaran V, Ikeda H, Bruce AT, White JM, Swanson PE, Old LJ and Schreiber RD: IFNgamma and lymphocytes prevent primary tumour development and shape tumour immunogenicity. Nature 410: 1107-1111, 2001.

28. Raez LE, Fein S and Podack ER: Lung cancer immunotherapy. Clin Med Res 3: 221-228, 2005.

29. Hirschowitz EA, Foody T, Kryscio R, Dickson L, Sturgill J and Yannelli J: Autologous dendritic cell vaccines for non-small-cell lung cancer. J Clin Oncol 22: 2808-2815, 2004. 